\title{
QoS-Based Dynamic Channel Allocation for GSM/GPRS Networks
}

\author{
Jun Zheng ${ }^{1}$ and Emma Regentova ${ }^{2}$ \\ 1 Department of Computer Science, \\ Queens College - The City University of New York, USA \\ zheng@cs.qc.edu \\ 2 Deaprtment of Electrical and Computer Engineering, \\ University of Nevada, Las Vegas, USA \\ regent@egr.unlv.edu
}

\begin{abstract}
Efficient channel allocation is important for meeting the quality of service (QoS) requirements of both GSM voice calls and GPRS packets in integrated GSM/GPRS networks. In this paper, we propose a new dynamic channel allocation scheme with guard channel, channel deallocation/re-allocation for voice call and packet queue for GSM/GPRS networks. An analytic model with general GPRS channel requirement is developed to evaluate the performance of the proposed scheme. Numerical results demonstrate that the scheme can adapt to different QoS requirements of the system by adjusting the number of guard channels and the size of packet queue. Compared to some conventional schemes, the proposed scheme achieves better performance of QoS provisioning.
\end{abstract}

\section{Introduction}

General Packet Radio Service (GPRS) utilizes the existing GSM network infrastructure to provide end-to-end packet-switched service [1]. To ensure the required quality of service (QoS) of the voice and data in the integrated GSM/GPRS networks, the channel allocation scheme has to optimally use the scarce radio resource. Dynamic resource allocation is believed to be a judicious solution for the problem.

Dynamic channel allocation in GSM/GPRS networks has received a considerable attention in recent studies. For example, Lin et al. [2] investigated four resource allocation algorithms, i.e., fix resource allocation (FRA), fix resource allocation with queue capability (FRAQ), dynamic resource allocation (DRA) and dynamic resource allocation with queue capability (DRAQ). In the dynamic schemes, partial resources can be allocated to the GPRS packet request. This kind of dynamic allocation can substantially reduce the GPRS dropping probability. It is also indicated that the voice queuing mechanism could significantly lower the GSM voice call incompletion probability. In [3], Lin et al. studied the buffering mechanisms for the dynamic resource allocation by employing both the voice queue and the packet queue. The results have demonstrated that packet 
queuing greatly reduces the GPRS packet dropping probability while the performance of the voice call slightly degrades. Chen et al. [4] proposed a channel de-allocation scheme (DAS) which decreases the GSM voice call incompletion probability by de-allocating a channel from on-going GPRS packet to new arrived GSM voice call if there is no free channel in the system. In [5], the authors employed both DAS and packet queue. It was shown that although the packet buffering mechanism can lessen the packet dropping probability, it increases the voice call incompletion probability even with the DAS in use. Recently, Zhang and Soong [6] introduced a channel re-allocation scheme (RAS) that re-allocates released idling channels to the GPRS data using partial resources. Results confirm that RAS sharply decreases the GSM voice call incompletion probability at the expense of the slight increment of the GPRS packet dropping probability.

In all the above studies, the new and handoff GSM voice calls are not differentiated. However, in the real systems, handoff calls always have a higher priority than new voice calls. This is because termination of a former is more noticeable, hence more annoying for users than blocking of a new call. In this paper, we enforce the priority of handoff voice calls, and propose a dynamic channel allocation scheme with guard channel, channel de-allocation/re-allocation for voice call and packet queue. In the proposed scheme, the guard channel is for lowering the dropping probability of handoff calls. Channel de-allocation/re-allocation for voice call lessens both the new and handoff voice call blocking probabilities and the packet queuing is used for reducing the packet dropping probability. By dynamically adjusting the guard channel capacity and the packet queue size, the scheme can adapt to the QoS requirements of the system on the new/hadnoff voice call blocking probability and GPRS packet dropping probability.

To study the system performance under the proposed dynamic channel allocation scheme, we derive an analytic model. In contrast with the models in $[2][3][4][5]$ that have adopted a specific maximum numbers of channels for GPRS packet for the sake of analytical simplicity, we derive a model with generalized GPRS channel requirement based on the one presented in [6]. That offers more versatility to the performance analysis.

The rest of this paper is organized as follows. In the next section, we introduce the QoS-based dynamic channel allocation scheme with guard channel, channel de-allocation/re-allocation for voice call and packet queue. The analytical model for evaluating the performance of the proposed scheme is developed in Section 3. Numerical results are presented and discussed in Section 4, and followed are the conclusions drawn in Section 5.

\section{QoS-Based Dynamic Resource Allocation Scheme}

We consider the GSM/GPRS network is homogeneous such that we only need to analyze one cell case. Assume the base station (BS) of each cell has $C$ channels shared by GSM voice calls and GPRS data packets. The maximum number of channels for data packet transmission is $M$. A type- $m$ GPRS call is the GPRS packet transmitted using $m(m=1, \ldots, M)$ channels. Assume the number of free 
channels in the system is $C_{F}$ which equals to $C-n_{v n}-n_{v h}-\sum_{m=1}^{M} m n_{g_{m}}$, where $n_{v n}$ is the number of new voice calls in service, $n_{v h}$ is the number of handoff voice calls, $n_{g_{m}}$ is the number of type- $m$ ongoing GPRS packet transmissions. The proposed scheme reserves $g$ channels as guard channels only for serving handoff GSM voice calls. The remaining $(C-g)$ channels are shared by both new/handoff GSM voice calls and GPRS calls. The size of the packet queue is $B$, and the number of GPRS calls buffered in the packet queue is denoted as $n_{P Q}$.

The state of the system changes according to the arrival and the completion of new/handoff GSM voice call and GPRS call. For the new GSM voice call arrival, the call will be served if $C_{F}>g$. The handoff voice call will be served if $C_{F}>0$. Upon arrival of GPRS packet, $M$ channels are allocated if $C_{F} \geq M+g$. If $g<C_{F}<M+g,\left(C_{F}-g\right)$ channels are allocated to the GPRS call. If $C_{F} \leq g$ and the number of buffered GPRS calls is less than $B$, the GPRS call will be buffered in the packet queue. Otherwise, the GPRS packet is dropped. For a new arrived voice call, if $C_{F} \leq g$, channel de-allocation allows one channel from an ongoing type- $m(m>1)$ GPRS call (or degradable GPRS call) to service the arrived voice call. If no degradable GPRS call exists, the arrived new voice call will be blocked. For handoff voice call, if $C_{F}=0$, channel de-allocation will be applied to service the call if there exists a degradable GPRS call in the system. Otherwise, the handoff voice call will be forced to terminate. Note that the channel is deallocated from the GPRS call with the highest number of channels, e.g. a type- $m$ GPRS call can be degraded if there is no type- $q(q=m+1, \ldots, M-1, M)$ GPRS call in the BS. Upon the channel release due to the GSM voice call termination or handoff or completion of the GPRS packet transmission, if there are degraded GPRS calls in the system and no GPRS call buffered in the packet queue, the released channels will be re-allocated to upgrade the transmission of these calls. The re-allocation is performed using "worst degraded first upgrading" policy [6]. That is, a type- $m$ GPRS call can be upgraded if all type- $q(q=1,2, \ldots, m-1)$ GPRS calls have been upgraded to type- $M$. If there are GPRS calls buffered in the packet queue, the released channels are used to service the buffered GPRS call instead of re-allocating for degraded on-going GPRS calls.

To demonstrate the performance of the proposed scheme, we compare it with three other dynamic resource allocation schemes described below.

(1) Scheme 1 (referred as DRA1) is the same as the DRA proposed in [2];

(2) Scheme 2 (referred as DRA2) is the same as the scheme with channel deallocation/re-allocation for voice call in [6];

(3) Scheme 3 (referred as DRA3) is similar to the proposed scheme except the channel de-allocation/re-allocation for voice call is not applied.

\section{Analytic Model}

For the purpose of performance analysis of the proposed dynamic channel allocation scheme, we develop an analytic model. We assume that the new GSM voice call, the handoff GSM voice call and the GPRS packet all follow the Poisson 
process with arrival rate $\lambda_{v n}, \lambda_{v h}$ and $\lambda_{g}$, respectively. The voice call holding time and the cell residence time are assumed to be exponentially distributed with means $1 / \mu_{c h}, 1 / \mu_{c r}$, respectively. Then the channel holding time for voice call is exponentially distributed with rate $\mu_{v}=\mu_{c h}+\mu_{c r}$. If one channel is allocated to a GPRS packet, the packet transmission is assumed to follow the exponential distribution with mean $1 / \mu_{g}$. Thus the mean packet transmission time is also exponentially distributed with mean $1 / m \mu_{g}$ when $m$ channels are allocated. The handoff of the GPRS packet transmission is not considered, because the transmission time of individual packet is negligible, and transmission is completed before the handoff procedure starts [3].

The dynamic resource allocation scheme is modeled as a $(M+3)$-dimension Markov process. A state in this process is denoted as $s=\left(n_{v n}, n_{v h}, n_{g_{1}}, n_{g_{2}}, \ldots\right.$, $\left.n_{g_{M-1}}, n_{g_{M}}, n_{P Q}\right)$. The state space $\boldsymbol{S}$ of the Markov process is given by

$$
\begin{aligned}
\boldsymbol{S}= & \left(s=\left(n_{v n}, n_{v h}, n_{g_{1}}, n_{g_{2}}, \ldots, n_{g_{M-1}}, n_{g_{M}}, n_{P Q}\right) \mid\right. \\
& 0 \leq n_{v n}+n_{v h}+\sum_{k=1}^{M} k n_{g_{k}} \leq C-g, 0 \leq n_{v n} \leq C-g, \\
& \left.0 \leq n_{v h} \leq C-g, 0 \leq n_{g_{k}} \leq\left\lfloor\frac{C-g}{k}\right\rfloor(k=1,2, \ldots, M), n_{P Q}=0\right) \\
& \bigcup\left(s=\left(n_{v n}, n_{v h}, n_{g_{1}}, n_{g_{2}}, \ldots, n_{g_{M-1}}, n_{g_{M}}, n_{P Q}\right) \mid\right. \\
& 0 \leq n_{v n}+\sum_{k=1}^{M} k n_{g_{k}} \leq C-g, C-g<n_{v n}+n_{v h}+\sum_{k=1}^{M} k n_{g_{k}} \leq C, \\
& \left.0 \leq n_{v n} \leq C-g, 0 \leq n_{v h} \leq C, 0 \leq n_{g_{k}} \leq\left\lfloor\frac{C}{k}\right\rfloor(k=1,2, \ldots, M), n_{P Q} \geq 0\right)
\end{aligned}
$$

Denote the steady state probability for state $s$ as $\pi_{s}$. For all states $s \in \boldsymbol{S}$, $\sum_{s \in S} \pi_{s}=1$. To find the steady state probability matrix $\boldsymbol{\Pi}$, we need to obtain the generator matrix $\mathbf{Q}=\left[q_{s \rightarrow s^{\prime}}\right]_{s \in S, s^{\prime} \in S}$, where $q_{s \rightarrow s^{\prime}}\left(s \in \boldsymbol{S}, s^{\prime} \in \boldsymbol{S}\right)$ is the transition rate from state $s=\left(n_{v n}, n_{v h}, n_{g_{1}}, n_{g_{2}}, \ldots, n_{g_{M-1}}, n_{g_{M}}, n_{P Q}\right)$ to state $s^{\prime}=\left(n_{v n}^{\prime}, n_{v h}^{\prime}, n_{g_{1}}^{\prime}, n_{g_{2}}^{\prime}, \ldots, n_{g_{M-1}}^{\prime}, n_{g_{M}}^{\prime}, n_{P Q}^{\prime}\right)$.

Given below are some definitions for deriving the generator matrix $\mathbf{Q}$.

$-I_{A}$ : The indicator function which equals to $1(0)$ when the event $A$ is true (false).

$-n_{g_{\alpha}}$ : The first non-zero value in the sequence $\left(n_{g_{M}}, n_{g_{M}-1}, \ldots, n_{g_{2}}\right)$, where $\alpha=\max \left(m \mid n_{g_{m}}>0, m=M, M-1, \ldots, 2\right)$. For $\left(n_{g_{M}}, n_{g_{M}-1}, \ldots, n_{g_{2}}\right) \equiv 0$, $\alpha$ is set to -1 . Thus if $\alpha \geq 2$, there exists a degradable GPRS call.

$-n_{g_{\beta}}$ : The first non-zero value in the sequence $\left(n_{g_{1}}, n_{g_{1}}, \ldots, n_{g_{M-1}}\right)$, where $\beta=\min \left(k \mid n_{g_{k}}>0, k=1,2, \ldots, M-1\right)$. For $\left(n_{g_{1}}, n_{g_{2}}, \ldots, n_{g_{M-1}}\right) \equiv 0, \beta$ is set to -1 . Thus if $\beta \geq 0$, there exists a degraded GPRS call.

- $\mathbf{M}$ : The state space of the GPRS call type, $\mathbf{M}=(1,2, \ldots, M-1, M)$.

To obtain the transition rate $q_{s \rightarrow s^{\prime}}$, we consider two cases according to the arrival and completion events of new/handoff GSM voice call and GPRS call.

\section{Case 1: New/handoff GSM voice call and GPRS call arrival}

The transitions from state $s$ to all possible next state $s^{\prime}$ for new/handoff GSM voice call and GPRS call arrival are shown in Table 1, where the first column shows the changes between $s$ and $s^{\prime}$ followed with the condition for the transitions, transition rates and the event related to the transition. 


\section{Case 2: New/handoff GSM voice call and GPRS call completion}

Table 2 shows the state transitions under new/handoff GSM voice call and GPRS call completion. Here we discuss in detail the case of type- $m$ GPRS call completion and $n_{P Q}=0$ that channel re-allocation can be used by allocating the released $m$ channels to upgrade degraded GPRS calls in the system. In this case, upon the type- $m$ GPRS call completion, the new state becomes $s_{1}$ $=\left(n_{v n}^{*}, n_{v h}^{*}, n_{g_{1}}^{*}, \ldots, n_{g_{m}}^{*}, \ldots, n_{g_{M}}^{*}, n_{P Q}^{*}\right)$, where $n_{v n}^{*}=n_{n v}, n_{v h}^{*}=n_{v h}, n_{g_{m}}^{*}=$ $n_{g_{m}}-1, n_{g_{k}}^{*}=n_{g_{k}}(k \in \mathbf{M}-\{m\}), n_{P Q}^{*}=n_{P Q}$. An index $\theta(1 \leq \theta \leq M-1)$ is introduced such that

$$
\sum_{k=1}^{\theta-1} n_{g_{k}}^{*}(M-k) \leq m \leq \sum_{k=1}^{\theta} n_{g_{k}}^{*}(M-k) .
$$

Inequality (2) implies that when a type- $m$ GPRS call leaves the BS, all the type- 1 to type- $(\theta-1)$ GPRS calls and some of the type- $\theta$ GPRS calls can be upgraded to type- $M$ GPRS calls. Denote

$$
\delta=\left\lfloor\frac{m-\sum_{k=1}^{\theta-1} n_{g_{k}}^{*}(M-k)}{M-\theta}\right\rfloor
$$

as the number of type- $\theta$ calls that can be upgraded to type- $M$ calls. $\delta_{r}=\delta-$ $\delta(M-\theta)$ is the number of channels that can upgrade a type- $\theta$ call to type$\left(\theta+\delta_{r}\right)$. If $\sum_{k=1}^{M-1} n_{g_{k}}^{*}(M-k) \leq m$ which means that all the type-1 to type$(M-1)$ calls can be upgraded to type- $M$ calls, we set $\theta$ to $M$. If $\beta=0$ which means that there is no data call in the BS or all the data calls are type- $M$, we set $\theta$ to -1 .

The transition rate $q_{s \rightarrow s}$ can be obtained as

$$
q_{s \rightarrow s}=-\sum_{s^{\prime} \neq s, s \in S, s^{\prime} \in S} q_{s \rightarrow s^{\prime}}
$$

Table 1. Transitions from $s$ to $s^{\prime}$ for new/handoff GSM voice call and GPRS call arrival. A: New GSM voice call, B: Handoff GSM voice call, C: Type-m GPRS call.

\begin{tabular}{|c|c|c|c|}
\hline Next state $s^{\prime}$ & Condition & Rate & Event \\
\hline$n_{v n}^{\prime}=n_{v n}+1$ & $C_{F}>g$ & $\lambda_{v n}$ & $\mathrm{~A}$ \\
\hline $\begin{array}{c}n_{v n}^{\prime}=n_{v n}+1, n_{g_{\alpha}-1}^{\prime}=n_{g_{\alpha}-1}+1, \\
n_{g_{\alpha}}^{\prime}=n_{g_{\alpha}}-1\end{array}$ & $C_{F} \leq g$ and $\alpha \geq 2$ & $\lambda_{v n}$ & $\mathrm{~A}$ \\
\hline$n_{v h}^{\prime}=n_{v h}+1$ & $C_{F}>0$ & $\lambda_{v h}$ & $\mathrm{~B}$ \\
\hline $\begin{array}{c}n_{v h}^{\prime}=n_{v h}+1, n_{g_{\alpha}-1}^{\prime}=n_{g_{\alpha}-1}+1, \\
n_{g_{\alpha}}^{\prime}=n_{g_{\alpha}}-1\end{array}$ & $C_{F}=0$ and $\alpha \geq 2$ & $\lambda_{v h}$ & $\mathrm{~B}$ \\
\hline$n_{g_{M}}^{\prime}=n_{g_{M}}+1$ & $C_{F} \geq M+g$ & $\lambda_{g}$ & $\mathrm{C}$ \\
\hline$n_{g_{C_{F}-g}}^{\prime}=n_{g_{C_{F}-g}}+1$ & $g<C_{F}<M+g$ & $\lambda_{g}$ & $\mathrm{C}$ \\
\hline$n_{P Q}^{\prime}=n_{P Q}+1$ & $C_{F} \leq g, B>0$ and $n_{P Q}<B$ & $\lambda_{g}$ & $\mathrm{C}$ \\
\hline
\end{tabular}


Table 2. Transitions from $s$ to $s^{\prime}$ for new/handoff GSM voice call and GPRS call completion. D: New GSM voice call, E: Handoff GSM voice call, F: Type- $m$ GPRS call.

\begin{tabular}{|c|c|c|c|}
\hline Next state $s^{\prime}$ & Condition & Rate & Event \\
\hline $\begin{aligned} n_{v n}^{\prime}= & n_{v n}-1, n_{g_{1}}^{\prime}=n_{g_{1}}+1, \\
& n_{P Q}^{\prime}=n_{P Q}-1\end{aligned}$ & $n_{P Q}>0$ & $n_{v n} \mu_{v}$ & $\mathrm{D}$ \\
\hline $\begin{array}{c}n_{v n}^{\prime}=n_{v n}-1, n_{g_{\beta}}^{\prime}=n_{g_{\beta}}-1, \\
n_{g_{\beta}+1}^{\prime}=n_{g_{\beta}+1}+1\end{array}$ & $n_{P Q}=0$ and $\beta>0$ & $n_{v n} \mu_{v}$ & $\mathrm{D}$ \\
\hline$n_{v n}^{\prime}=n_{v n}-1$ & $n_{P Q}=0$ and $\beta=-1$ & $n_{v n} \mu_{v}$ & $\mathrm{D}$ \\
\hline $\begin{aligned} n_{v h}^{\prime}= & n_{v h}-1, n_{g_{1}}^{\prime}=n_{g_{1}}+1, \\
& n_{P Q}^{\prime}=n_{P Q}-1\end{aligned}$ & $C_{F} \geq g$ and $n_{P Q}>0$ & $n_{v h} \mu_{v}$ & $\mathrm{E}$ \\
\hline $\begin{array}{c}n_{v h}^{\prime}=n_{v h}-1, n_{g_{\beta}}^{\prime}=n_{g_{\beta}}-1, \\
n_{g_{\beta}+1}^{\prime}=n_{g_{\beta}+1}+1\end{array}$ & $C_{F} \geq g, n_{P Q}=0$ and $\beta>0$ & $n_{v h} \mu_{v}$ & $\mathrm{E}$ \\
\hline$n_{v h}^{\prime}=n_{v h}-1$ & $C_{F} \geq g, n_{P Q}=0$ and $\beta=-1$ & $n_{v h} \mu_{v}$ & $\mathrm{E}$ \\
\hline$n_{v h}^{\prime}=n_{v h}-1$ & $C_{F}<g$ & $n_{v h} \mu_{v}$ & $\mathrm{E}$ \\
\hline$n_{P Q}^{\prime}=n_{P Q}-1$ & $n_{P Q}>0$ & $n_{g_{m}} m \mu_{g}$ & $\mathrm{~F}$ \\
\hline$n_{g_{m}}^{\prime}=n_{g_{m}}-1$ & $n_{P Q}=0$ and $\theta=-1$ & $n_{g_{m}} m \mu_{g}$ & $\mathrm{~F}$ \\
\hline $\begin{array}{c}n_{g_{k}}^{\prime}=0(k=1,2, \ldots, \theta-1), \\
n_{g_{\theta}}^{\prime}=n_{g_{\theta}}-\delta-1, \\
n_{g_{\theta+\delta_{r}}}^{\prime}=n_{g_{\theta+\delta_{r}}}+1, \\
n_{g_{M}}^{\prime}=n_{g_{M}}+\sum_{k=1}^{\theta-1} n_{g_{k}}+\delta\end{array}$ & $n_{P Q}=0$ and $1 \leq \theta \leq M-1$ & $n_{g_{m}} m \mu_{g}$ & $\mathrm{~F}$ \\
\hline $\begin{array}{c}n_{g_{k}}^{\prime}=0(k \in \mathbf{M}-\{M\}) \\
n_{g_{M}}=\sum_{k=1}^{M} n_{g_{k}}-1\end{array}$ & $n_{P Q}=0$ and $\theta=M$ & $n_{g_{m}} m \mu_{g}$ & $\mathrm{~F}$ \\
\hline
\end{tabular}

From Table 1 and 2 and Eq. (3), we can derive the generator matrix $\mathbf{Q}$ for the $(M+3)$-dimension Markov chain. To obtain the steady-state probability matrix $\boldsymbol{\Pi}$, we need to solve the linear equation $\boldsymbol{\Pi} \mathbf{e}=1$ and $\mathbf{\Pi Q}=0$, where $\mathbf{e}$ is a unary column vector. This is done using a numerical method introduced in [7].

Knowing the steady-state probability $\pi_{s}$ of the Markov chain, we can calculate the handoff GSM voice call arrival rate as

$$
\lambda_{v h}=\sum_{s \in S}\left(n_{v n}+n_{v h}\right) \pi_{s} \mu_{c r}
$$

Since the steady-state probability $\pi_{s}$ and the handoff call arrival rate $\lambda_{v h}$ are mutually related, an iterative algorithm is applied to compute $\pi_{s}$ and $\lambda_{v h}$ as in $[8]$.

To measure the performance of the proposed scheme, we use the following performance metrics - new GSM voice call blocking probability $P_{v n}$, handoff GSM voice call forced termination probability $P_{v h}$, GPRS packet dropping probability $P_{g}$ and channel utilization $u$.

The new GSM voice call will be blocked if the number of free channels $C_{F} \leq g$ and the GPRS calls in the BS are all type-1. Then, the new GSM voice call blocking probability $P_{v n}$ is represented as 


$$
P_{v n}=\sum_{n_{P Q}=0}^{B} \sum_{\substack{n_{v n}+n_{v h}+n_{g_{1}} \geq C-g, n_{g_{k}}=0(k \in \mathbf{M}-\{1\}), s \in S}} \pi_{s}
$$

The handoff GSM voice call will be forced to terminate if the number of free channels $C_{F}=0$, and the GSM calls in the BS are all of type-1. The handoff GSM voice call dropping probability $P_{v h}$ is obtained as

$$
P_{v h}=\sum_{n_{P Q}=0}^{B} \sum_{\substack{n_{v n}+n_{v h}+n_{g_{1}}=C, n_{g_{k}}=0(k \in \mathrm{M}-\{1\}), s \in S}} \pi_{s}
$$

The GPRS packet will be dropped if the number of free channels $C_{F} \leq g$ and the packet queue is full. The GPRS packet dropping probability $P_{g}$ is then represented as

$$
P_{g}=\sum_{\substack{n_{v n}+n_{v h}+n_{g_{1}} \geq C-g, n_{P Q}=B, s \in S}} \pi_{s}
$$

The channel utilization $u$ can be expressed as

$$
u=\frac{\sum_{s \in S}\left(n_{v n}+n_{v h}+\sum_{k=1}^{M} k n_{g_{k}}\right) \pi_{s}}{C}
$$

It should be noted that the above-mentioned performance metrics are influenced by the number of guard channels $g$ and the packet queue size $B$. To measure the QoS of the system, we use a system award $Q$ which is expressed as

$$
Q=\alpha\left(1-P_{v n}\right)+\beta\left(1-P_{v h}\right)+\gamma\left(1-P_{g}\right)
$$

where $\alpha, \beta$ and $\gamma$ are weighting factors which indicate the contribution of $P_{v n}$, $P_{v h}$ and $P_{g}$ to the system's QoS, respectively. Notice that $\alpha+\beta+\gamma=1$. The weighting factors are determined by the system's overall revenue and service objectives. A larger $Q$ indicates higher performance of the scheme.

\section{Numerical Results}

Based on the derived analytic model, we can evaluate the performance of the proposed dynamic resource allocation scheme. We normalize the parameters $\lambda_{v n}$, $\lambda_{g}, \mu_{c r}$ and $\mu_{g}$ by $\mu_{c h}$ as done in [2][3]. The number of channels $C$ in the BS is assumed to be 7 .

Figures 1(a) through $1(\mathrm{~d})$ compare the performance of the proposed scheme with that of other three schemes under different GPRS traffic load $\rho_{g}$. The parameters are set as $M=2, B=4, g=1, \mu_{c h}=1 / 180, \mu_{c r}=0.2 \mu_{c h}, \mu_{g}=$ $100 \mu_{c h}$ and $\rho_{v}=2$. Fig. 1 (a) shows that DRA1 and DRA2 without packet queue capability have a higher $P_{g}$ compared to that of DRA3 and the proposed scheme 


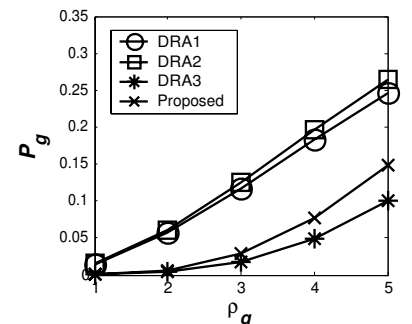

(a)

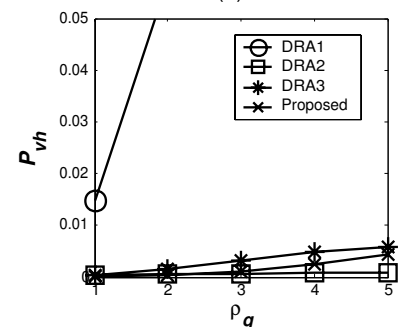

(c)

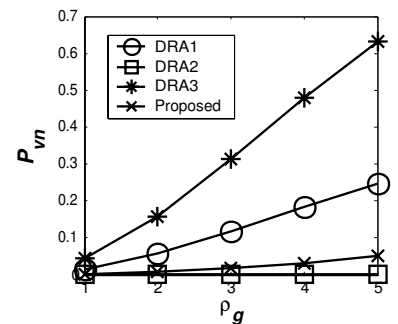

(b)

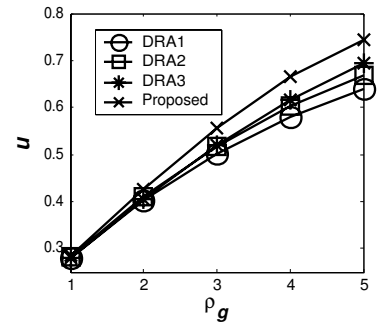

(d)

Fig. 1. Performance comparison for different schemes. (a) $P_{g}$, (b) $P_{v n}$, (c) $P_{v h}$, (d) $u$.

with packet queue. DRA3 has lower $P_{g}$ than that of the proposed scheme because DRA3 does not use channel de-allocation/re-allocation for voice calls. From Fig. 1(b), we can see that $P_{v n}$ is ranging from low to high for DRA2, the proposed scheme, DRA1 and DRA3. By using channel de-allocation/re-allocation for voice call, DRA2 and the proposed scheme achieves lower $P_{v n}$. Since the proposed scheme employs the packet queue and guard channels that results in higher $P_{v n}$, DRA2 outperforms the proposed scheme in terms of $P_{v n}$. DRA3 has the highest $P_{v n}$ because it uses the packet queue and guard channels without channel deallocation/re-allocation for voice call. Fig. 1(c) shows that $P_{v h}$ ranging from low to high are DRA2, the proposed scheme, DRA3 and DRA1. From Fig. 1(d), one can observe that the proposed scheme achieves the best channel utilization. Followed are DRA3, DRA2 and DRA1.

Figure 2(a) to 2(d) show the system award $Q$ as a function of $B$ and $g$ for different system QoS requirements. They correspond to four cases with varying weight factors for the system award $Q$ : (a) $\alpha=0.8, \beta=0.1$ and $\gamma=0.1$, (b) $\alpha=0.1, \beta=0.8$ and $\gamma=0.1$, (c) $\alpha=0.1, \beta=0.1$ and $\gamma=0.8$ and (d) $\alpha=$ $0.4, \beta=0.3$ and $\gamma=0.3$. Other parameters are set as $M=2, \mu_{c h}=1 / 180$, $\lambda_{v n}=2 \mu_{c h}, \lambda_{g}=200 \mu_{c h}, \mu_{c r}=0.4 \mu_{c h}, \mu_{g}=100 \mu_{c h}, 0 \leq g \leq 5,0 \leq B \leq 6$. For Fig. 2(a), we can find $g=0$ and $B=1$ produce the best value of $Q$. This is due to the fact that $P_{v n}$ is the most important factor in the system award $Q$ ( $\alpha=0.8$ ) and larger $g$ and $B$ will result in higher $P_{v n}$. In Fig. 2(b), the best $Q$ is achieved for $g=1$ and $B=3$. In this case, the system emphasizes $P_{v h}(\beta=0.8)$ and one channel is reserved only for handoff voice calls. Fig. 2(c) shows the best $Q$ produced when $g=0, B=6$. In this case, the system prefers GPRS packet to GSM voice call $(\gamma=0.8)$ and a larger packet queue size is used to reduce 


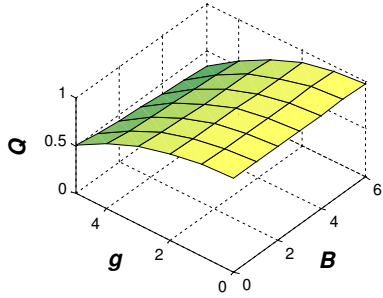

(a)

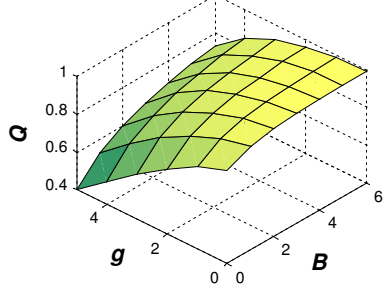

(c)

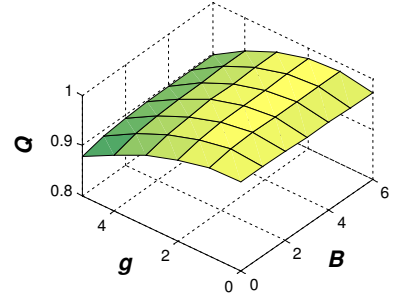

(b)

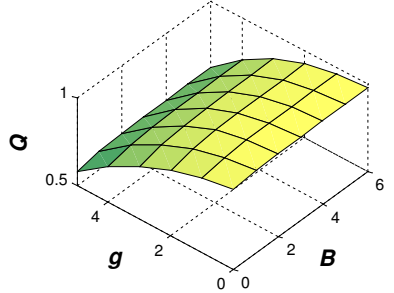

(d)

Fig. 2. System award $Q$ vs. $B$ and $g$ for(a) $\alpha=0.8, \beta=0.1$ and $\gamma=0.1$, (b) $\alpha=0.1$, $\beta=0.8$ and $\gamma=0.1$, (c) $\alpha=0.1, \beta=0.1$ and $\gamma=0.8$ and (d) $\alpha=0.4, \beta=0.3$ and $\gamma=0.3$

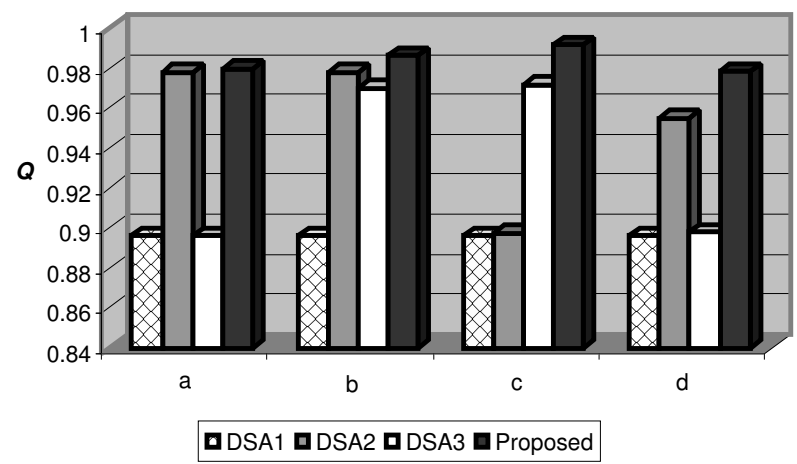

Fig. 3. System award comparison for different schemes. (a) $\alpha=0.8, \beta=0.1$ and $\gamma=$ 0.1 , (b) $\alpha=0.1, \beta=0.8$ and $\gamma=0.1$, (c) $\alpha=0.1, \beta=0.1$ and $\gamma=0.8$ and (d) $\alpha=$ $0.4, \beta=0.3$ and $\gamma=0.3$.

$P_{g}$. Finally, for the case shown in Fig. 2(d), the system has no preferences for new/handoff GSM voice call or GPRS packet, we can find that the best $Q$ is achieved by $g=0, B=4$.

We then compare the system award $Q$ of the four dynamic resource allocation schemes as shown in Fig. 3 for the same four cases and parameters as for Fig 2 . $Q$ values of DSA3 are obtained using the optimal combination of $g$ and $B$. The results demonstrate that the proposed scheme always outperforms other 
three reference schemes because it is furnished by the capability of adjusting the number of guard channels $g$ and packet queue size $B$ to meet the system's QoS requirements.

\section{Conclusion}

In the integrated GSM/GPRS networks, the GSM voice and the GPRS packet services use the same resources that makes the channel allocation a critical issue for the QOS provisioning for both. In this paper, we have discussed a new dynamic channel allocation scheme with guard channel, channel de-allocation/reallocation for voice call and packet queuing. The developed analytical model with generalized GPRS data channel requirement has allowed for evaluating the performance of the proposed scheme. The numerical results indicate that by adjusting the number of guard channels and the size of the packet queue, one can attain dynamical adaptation to different QoS requirements of the system.

\section{References}

1. Y.-B. Lin, H. C.-H. Rao and I. Chlamtac, "General Packet Radio Service (GPRS): architecture, interfaces and deployment," Wirel. Commun. Mob. Comput., vol. 1, pp. 77-92, 2001.

2. P. Lin and Y. -B. Lin, "Channel allocation for GPRS," IEEE Trans. on Vehicular Technology, vol. 50, no. 2, pp. 375-387, Mar. 2001.

3. P. Lin, "Channel allocation for GPRS with buffering mechanisms," Wireless Networks, vol. 9, pp. 431-441, 2003.

4. W. Y. Chen, J.-L. C. Wu and L. Lu, "Performance comparison of dynamic resource allocation with/without channel de-allocation in GSM/GPRS networks," IEEE Communications Letters, vol. 7, no. 1, pp. 10-12, Jan. 2003.

5. W. Y. Chen, J.-L. C. Wu and H. H. Liu, "Performance analysis of dynamic resource allocation with finite buffers in cellular networks," IEICE Trans. Fundementals, vol. E87-A, no. 7, pp. 1692-1699, July 2004.

6. Y. Zhang and B. Soong, "Performance evaluation of GSM/GPRS networks with channel re-allocation scheme," IEEE Communications Letters, vol. 8, no. 5, pp. 280-282, May 2004.

7. W. J. Stewart, Introduction to the Numerical Solution of Markov Chains, Princeton University Press, 1994.

8. Y. -B. Lin, "Performance modeling for mobile telephone networks," IEEE Network Magazine, vol. 11, no. 6, pp. 63-68, 1997. 